\title{
Microscopic Characterization of Uranium Ore Specimens Using the Peakaboo Analysis Platform
}

\author{
Stewart McIntyre ${ }^{1}$, Lisa Van Loon ${ }^{1}$, Nathaniel Sherry ${ }^{1}$, Michael Bauer ${ }^{1}$, Tom Kotzer ${ }^{2}$ and Neil Banerjee ${ }^{1}$ \\ ${ }^{1}$ Western University, London, Ontario, Canada, ${ }^{2}$ SRK Consulting, Saskatoon, Saskatchewan, Canada
}

\begin{abstract}
XRF maps of a uranium ore sample have been analyzed using software that allows the graphical spatial correlations of all detected elements to be measured. The association of uranium with arsenic, nickel and cesium was explored; all showed unique finely-granular microscopic patterns that could be used to assist the recovery of uranium and rare earths.
\end{abstract}

\section{Introduction}

Microscopic X-ray Fluorescence ( $\mu$ XRF) Spectroscopy has unique capabilities for mineral analysis. Chief among these is a high detection sensitivity to high $\mathrm{Z}$ elements, many of which are economically important such as uranium and precious metals. The most important role for $\mu \mathrm{XRF}$ analysis of a mineral ore is to measure associations between these elements and their mineral hosts. The measurements of such associations must be statistically valid. As such, a high density of spectral data is needed, covering a representative sampling area. This paper illustrates the use of a newly-developed software platform to characterize elemental associations in a uranium ore

\section{Experimental}

Powdered specimens of a uranium tailings from the Athabasca Basin in northern Saskatchewan were analyzed on the VESPERS bending magnet beamline at the Canadian Light Source. Samples were excited with a $10 \%$ band pass filter set at an energy of $17 \mathrm{keV}$. Spectral analysis was carried out using a quantitative version of the XRF analysis software Peakaboo 5.4 Q [1,2]. Using this software XRF intensities were corrected for differing elemental yields using the NIST SRM 610 [3] multi-element glass that was run during the same experimental session. This empirical method provides estimates of elemental compositions that have been shown to have an accuracy of a factor of 2-3.

\section{Results and Discussion}

Figure 1a shows a spatial map of the distributions of 5 major and minor elements within a $100 \mu \mathrm{m}$ field of the tailings sample. Low intensity pixels are filtered out, so that the shapes of crystalline minerals could be observed, such as the rectangular crystals. The small green region represents the highest concentration of uranium present within the map (estimated to be 7\%). An adjacent violet region has the highest lead concentration (estimated as 5\%). The average $U$ concentration within the entire map is estimated to be $0.5 \%$. Nickel and arsenic are major elements in the entire field of view and are particularly prevalent in the rectangular crystals within which their ratios vary (as denoted by the varying pink and violet colorations). Individual analyses along the sharp lower edge of the crystal at image center give an average $\mathrm{Ni} /$ As ratio of $1.7: 1$.

Figure $1 \mathrm{~b}$ is a correlation diagram displaying the relationships between $\mathrm{Ni}$ and As for all pixels in the map. The nickel and arsenic concentrations shown on each axis may differ by as much as a factor of 2 from the values shown, but the same relationships will exist. One correlation pattern of note is the linear relationship that begins at the origin. The pixels represented here are those where these two elements are present in a constant ratio and with no interactions with other elements. Most pixels however, show more complex 
relationships: the two heavily populated (darkened) regions represent pixels with different Ni/As ratios and with additional elements interacting. The latter conclusion is drawn from the fact that no extended linear relationship is observed.

Figure 2 shows the correlations between arsenic and uranium in the map shown in Figure 1a. The linear relationship beginning at the origin implies a uranium-arsenic association exclusive of other elements. Most uranium - arsenic associations occur within pixels with a uranium concentration 4-5 times higher, and likely involving other elements. The highest concentration of uranium, shown in the upper inset, is the same green-colored area shown in Figure 1a. In addition to arsenic, associations with silicon and iron were detected (not shown). The most common uranium-arsenic association in Figure 2 is shown in the lower inset. The uranium distribution covering most of the map is finely detailed and shows microscopic clusters of U-As associations.

\section{Conclusions}

This is a preliminary study that will require the use of additional reference materials to improve the level of confidence in the accuracy of the measurements. In addition, measurements using three coordinate scales are planned so that the nickel-arsenic-uranium relationship can be better understood.

\section{Acknowledgement}

The research described in this paper was performed at the Canadian Light Source, a national research facility of the University of Saskatchewan, which is supported by the Canada Foundation for Innovation (CFI), the Natural Sciences and Engineering Research Council (NSERC), the National Research Council (NRC), the Canadian Institutes of Health Research (CIHR), the Government of Saskatchewan, and the University of Saskatchewan. N.S. McIntyre is grateful to the Canadian Foundation for Innovation for funding to construct the VESPERS beamline. The authors acknowledge funding from NSERC and CANARIE.

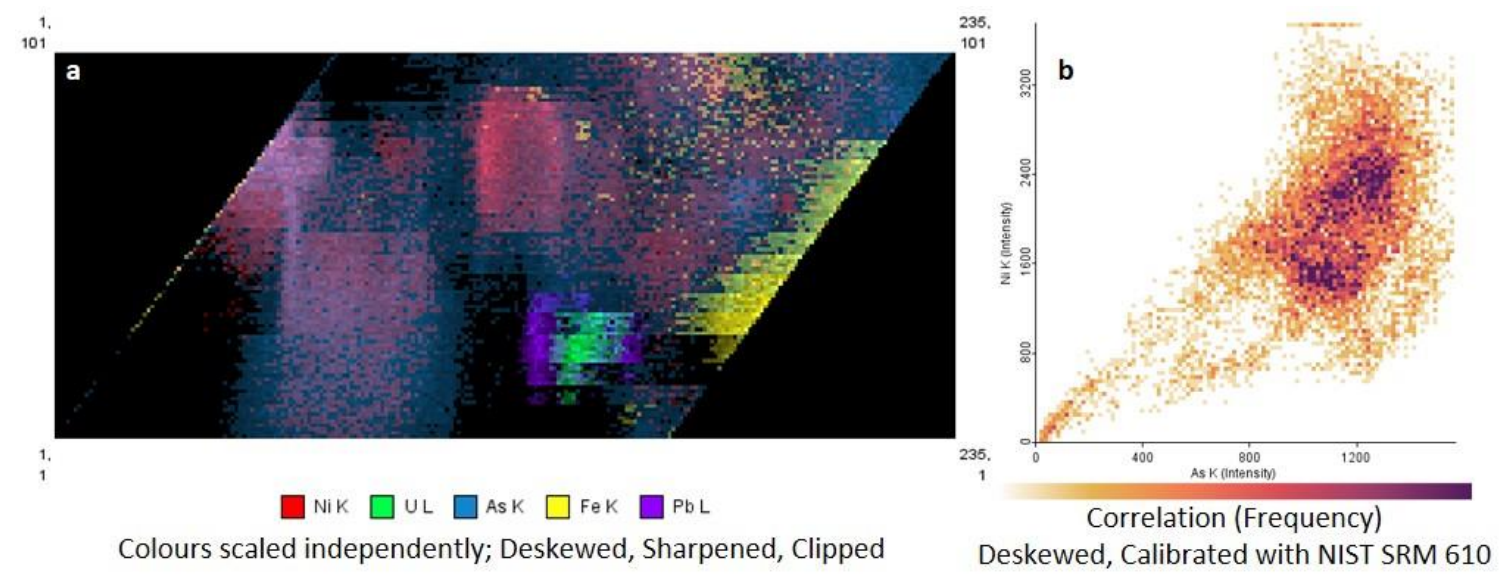

Figure 1. (a) Semi-quantitative XRF map of the distributions of the highest concentration elements in a uranium ore. The lowest intensities of each element were stripped from the image in order to clarify shapes of some of the crystals present. The smallest yellow crystals are iron sulfides and selenides. (b). Correlation plot of $\mathrm{Ni}$ vs As estimated concentrations for all pixels in the map. Each point represents a pixel where both $\mathrm{Ni}$ and As are detected. The measured concentrations of As and $\mathrm{Ni}$ are plotted on the $\mathrm{x}$ and y scales respectively. Where pixels have the same intensities, a thermal scale is used to represent the frequency of occurrence. 


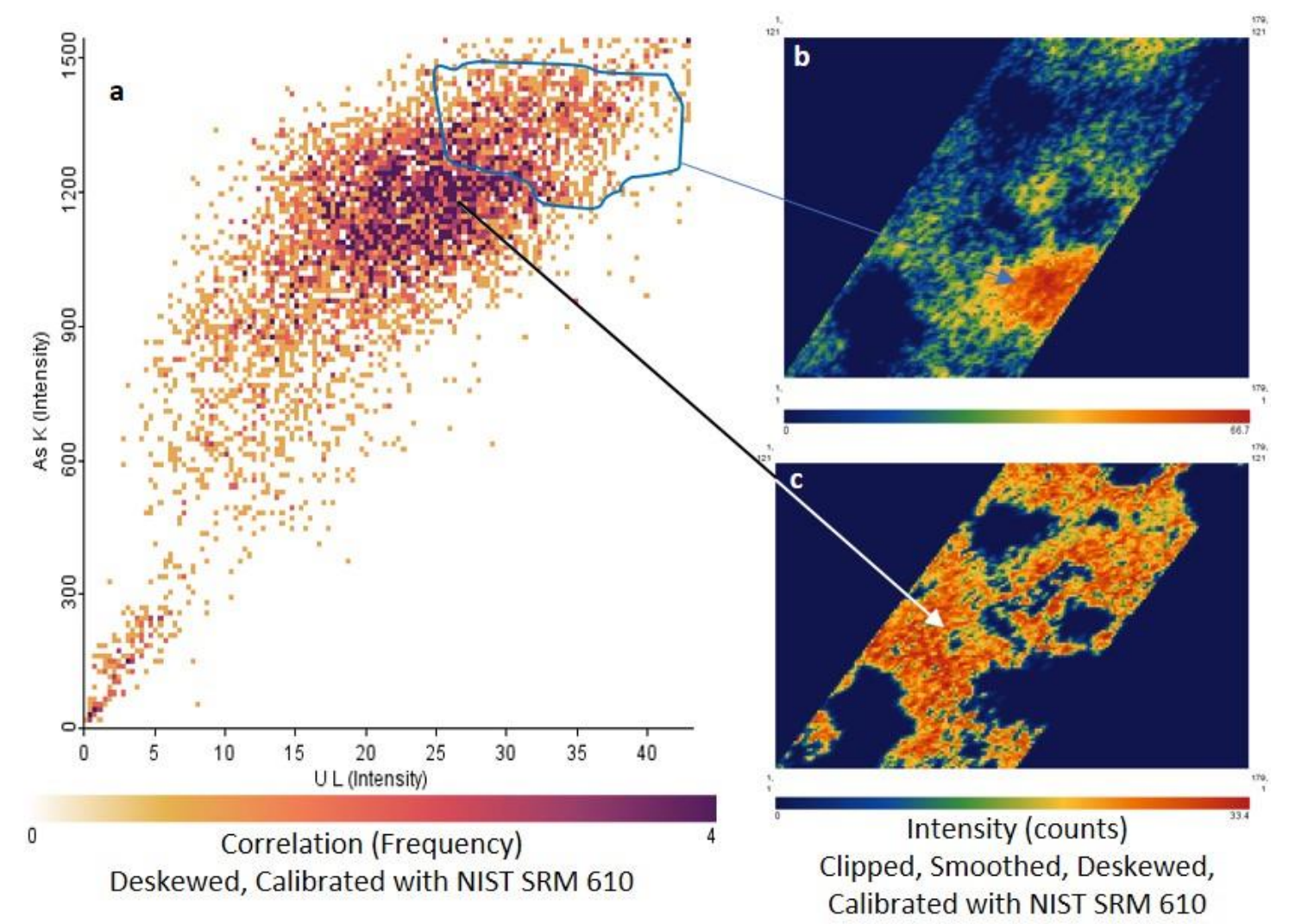

Figure 2. (a) Correlation plot of Arsenic vs. Uranium for all pixels from the map in Figure 1a.(b) The 2D $\mathrm{XRF}$ element map is reconstructed from the region in the correlation plot outlined in blue to show regions in the sample with the greatest U-As association. (c) The 2D XRF element map is reconstructed from the correlation plot in (a) to show regions in the sample with the most frequent association U-As association (darkest colour).

\section{References}

[1] Van Loon, L. L., McIntyre, N. S., Bauer, M., Sherry, N. S. A. \& Banerjee, N. R. (2019). Softw. Impacts. 2, 100010.

[2] http://www.peakaboo.org

[3] Wise, S. A. \& Watters Jr., R. L. (2012). Certificate of Analysis, Standard Reference Material® 610 Trace Elements in Glass. 